\title{
Effect of Fields Generated Through Wireless Power Transfer on Implantable Biomedical Devices
}

\author{
Nunzio Pucci*, Christopher H. Kwan*, David C. Yates*, Ahran D. Arnold ${ }^{\dagger}$, Daniel Keene ${ }^{\dagger}$, \\ Zachary I. Whinnett ${ }^{\dagger}$, Paul D. Mitcheson* \\ *Department of Electrical and Electronic Engineering \\ ${ }^{\dagger}$ National Heart \& Lung Institute \\ Imperial College London, United Kingdom \\ nunzio.pucci15@imperial.ac.uk
}

\begin{abstract}
This paper assesses the safety of pacemakers when exposed to the electromagnetic (EM) field generated by high frequency inductive power transfer (HF-IPT) systems. It includes both simulation and experimental results, showing temperature variations to ensure conformity with the EN standards, changes in detected lead impedance and determining whether EM field strength can affect the operating mode of the device. This is the first time the interaction between $6.78 \mathrm{MHz}, 100 \mathrm{~W}$ HF-IPT systems and pacemaker devices was tested up to distances of $5 \mathrm{~cm}$ to $10 \mathrm{~cm}$. Temporary decrease of detected lead's impedance and interruption of communications are the most relevant effects recorded through in-vitro tests. No permanent alteration of the device's operation was recorded, indicating good early stage evidence of safety for pacemaker users in proximity of this new emerging technology.
\end{abstract}

\section{INTRODUCTION}

In the past two decades the development of new technologies has increased concerns regarding safety of implantable devices such as pacemakers and ICDs. Induction hobs, airport scanners and MRI are just a few examples of technologies that could affect standard operating conditions of the implantable devices and represent a potential danger for users.

These problems have been tackled in different ways, leading to customized robust designs [1]:

- MRI-Safe devices;

- Adaptive blanking windows in the pacing algorithm;

- Replacement of unipolar leads with bipolar, for improved EMI rejection.

However, despite the large number of improvements implemented, these devices still need to be handled with particular care, especially under conditions in which strong electromagnetic fields are present [2]. With the large increase in popularity of electric vehicles, one technology that will likely spread within the next decade is kilowatt-level wireless chargers. The effects of wireless chargers have been tested for commercial applications and a wide amount of data is now available for such systems operating at $85 \mathrm{kHz}$ as part of the SAE J2954 wireless charging standard.

HF-IPT systems typically operate in the ISM band at 6.78 MHz, $13.56 \mathrm{MHz}$ and $27.12 \mathrm{MHz}$ leading to significant advantages compared to the low frequency IPT (LF-IPT) counterparts [5]. The differences between the two types of systems are relevant in terms of operating frequency, power levels, magnetic field strength and absence of a DC magnetic field.

Tests on pacemakers' compatibility with MRI [1] and LFIPT systems have been extensively conducted during the last two decades, however HF-IPT systems are still lacking conclusions on compatilibility from a structured safety assessment.

While the main focus of our analysis is avoiding general HF-IPT systems adversely affecting biomedical devices, HFIPT technology may also enable charging of biomedical devices themselves, at higher power levels. Battery longevity is a problem associated with permanent pacemakers (PPM) and implantable cardioverter-defibrillators (ICDs). Battery replacement is typically necessary after several years of PPM/ICD use (3-15 years depending on power consumption by the individual device). Battery replacement is a surgical procedure that carries a risks of infection and bleeding, both of which can be life threatening and the risk increases with each successive battery replacement.

One solution to the problem would be rapid, intermittent inductive charging of the device, completely eliminating the risks associated with surgical procedures. Similar applications would be found for other biomedical devices including lifesaving left ventricular assist devices (LVADs) [3], although one of the current difficulties that come with inductive charging is device interoperability in patients with multiple implants.

The results used for evaluation include CST Studio Suite simulations for temperature safety assessments. The first part of the paper compares the obtained simulation results with the Standards for Safety of Implantable Devices stated in EN 45502-1:2015 [4] under different simulating conditions.

The in-vitro testing results in Section $\mathrm{V}$ were obtained at Hammersmith Hospital, London. The tests replicate an environment in which the device is exposed to the HF magnetic field generated through the wireless charger, assessing the response of the different operating modes of a pacemaker device.

\section{RECORDED ISSUES RELATED TO THE TOPIC}

There is a wide variety of technologies able to affect the operating modes of a pacemaker, or even damage the circuitry of the device. Most of the effects are temporary, especially the ones related to adaptive pacing and sensing algorithms. 
However, extended exposure can be fatal. Some of the most dangerous consequences occur due to faults in the sensing circuit [2].

The sensing fault most likely to have fatal consequences is pacing inhibition. This usually happens when waves within $5 \mathrm{~Hz}$ are sensed as cardiac activity by the device. The active filters on the input side of the device cannot reject frequencies in this range since they are too close to potentially normal heart rates. Proper pacemaker function involves inhibition of pacing by sensed heartbeats for several reasons: facilitating natural, unpaced heartbeats, minimizing power usage and (most importantly from a safety perspective) avoiding pacing during a normal heart beat, which could otherwise trigger a fatal rhythm disorder. Inappropriate inhibition occurs when low frequency waves originating from non-cardiac sources of electrical signals are sensed as normal cardiac activity, thereby suppressing pacing in accordance with normal pacemaker function described above. Some patients are entirely dependent on pacing and inhibition of ventricular pacing is likely to be fatal in such cases. In rare cases MRI generated fields have been falsely sensed as atrial activity resulting in inappropriately triggered rapid ventricular pacing, which can not only cause uncomfortable palpitations (awareness of heartbeat) but also be life threatening in patients with already impaired cardiac function.

ICDs have a unique consequence of inappropriate EMI sensing. ICDs are designed to deliver high energy shocks to the heart if a life-threatening rhythm disorder (arrhythmia) is detected. However if EMI is falsely detected as an arrhythmia, shocks are delivered to an otherwise perfectly well patient. These shocks can be life threatening and worsen heart function but also have severe psychological consequences for the sufferer of inappropriate shocks. The introduction of bipolar leads, where the anode and the cathode for the pace/sense circuit are closely spaced within the heart, has improved device safety by reducing the incidence of inappropriate EMI sensing, but the problem has not been eliminated and sometimes unipolar sensing is required. Strong magnetic field exposure for extended periods can still result in inappropriate triggering and inhibition of pacing; many cases of inhibition due to MRI scanners have been reported even in the era of bipolar sensing. EMI-induced increases in lead impedance could result in higher energy requirement to achieve successful stimulation of cardiac tissue for successful pacing and thus lead to a failure to pace if the energy requirement exceeds the programmed energy output, which would be fatal in pacing-dependent patients.

Other faults recorded (mostly non-lethal) are resets, and temporary alterations of the adaptive blanking window for pacing. In the conducted in-vitro tests it was not possible to assess potential inhibition and effects on the adaptive blanking windows as this procedure would require detection of real cardiac signals, implying the need of specific in-vivo tests.

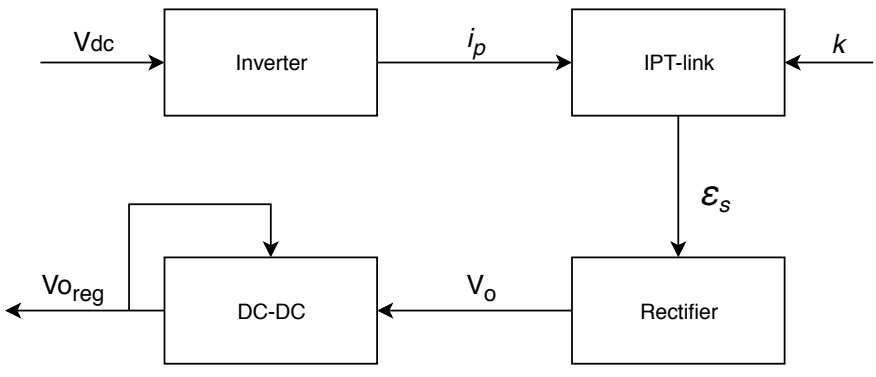

Fig. 1. Block Diagram IPT System

\section{Assessment METHOD}

The technology used consists of the transmitter coil of a 6.78 MHz IPT system for electric scooter charging (Fig. 1) [5]. The coil current is between $10 \mathrm{~A}$ and $20 \mathrm{~A}$; these amplitudes allow power transfer to the scooter of between $100 \mathrm{~W}$ and $600 \mathrm{~W}$.

The pacemaker type analysed for simulations is a singlechamber device using a bipolar-type lead.

The CST simulations are structured to perform an evaluation of the different changes in temperature due to variations in the coil's current (between $10 \mathrm{~A}$ and $20 \mathrm{~A}$ ) and distance (between $30 \mathrm{~cm}$ and $2 \mathrm{~m}$ ), showing the average case scenario in which a user walks close to the IPT system, and the worst case scenario in which the user faints next to the coil.

The experiments undertaken at Hammersmith Hospital replicate the same conditions analysed in the simulation environment (varying current and distance) and use the observed modes of operation that the implantable device enters as a benchmark for safety assessment.

The aim is to assess the potential dangers posed by HF-IPT systems and the levels of magnetic field strength at which they would occur.

\section{Simulations' RESUlts}

The first simulations stage shows positive results with respect to the temperature simulations.

When the pacemaker complete with lead is placed in front of the IPT coil (worst case scenario in which a pacemaker user faints next to the coil), the effects on the lead are small, mainly occurring in proximity of the external conductor, where the induced current is the highest. EN limits for temperature variations apply for surfaces in contact with the body.

Fig. 2 summarizes the effects of 60 seconds of exposure. The temperature variation, $\Delta T$ is less than $0.01{ }^{\circ} \mathrm{C}$, which proves to be within the European Standard limits $\left(\Delta T_{\mathrm{MAX}}\right.$ $=2{ }^{\circ} \mathrm{C}$ with respect to body temperature), meaning that even for worst case conditions temperature variation is not an issue when the exposure time is limited.

\section{IN-VITRO TESTING}

In-vitro testing of pacemaker devices was performed at Hammersmith Hospital, London, confirming initial hypothesis 


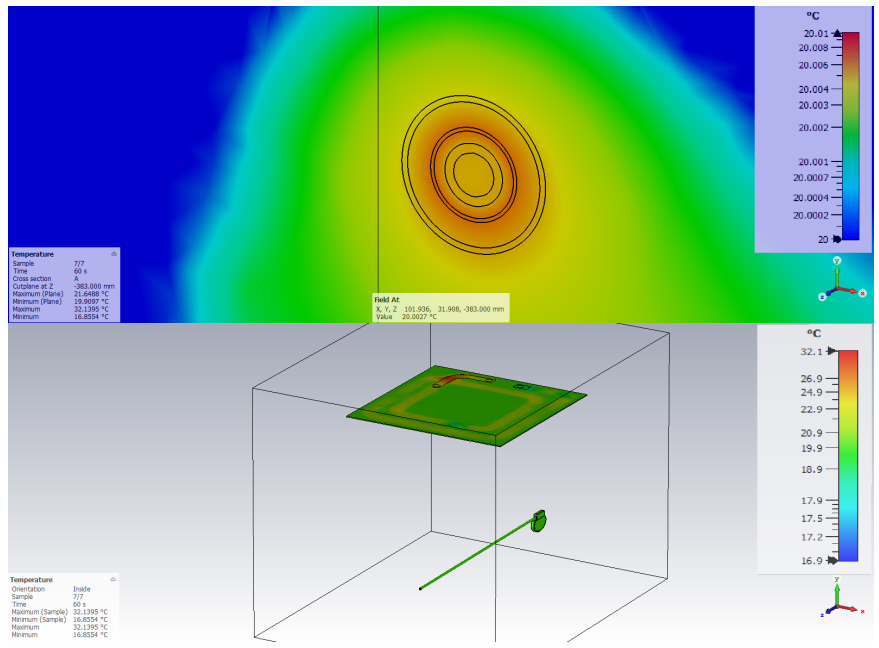

Fig. 2. Temperature Simulations, 60 seconds exposure, distance $=30 \mathrm{~cm}$

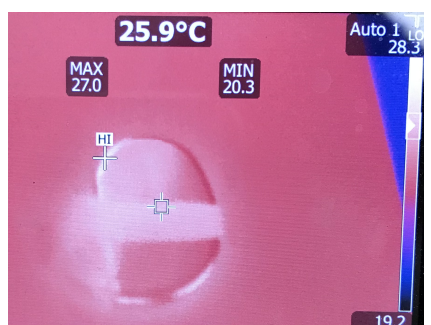

(a) $t=0$

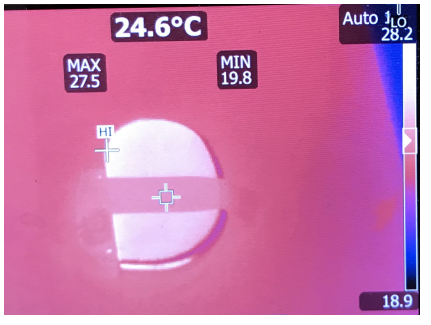

(b) $t=300 \mathrm{~s}$
Fig. 3. Effects of $300 \mathrm{~s}$ exposure on heating

made during the simulation stage and leading to new findings about the interaction between IPT coils and implantable biomedical devices.

\section{A. Technology used}

- Lead: Boston Scientific Ingevity MRI Pacing Lead 7741 $(52 \mathrm{~cm})$

- Pacemaker: Medtronic Ensura SR MRI SureScan

- Interrogator: Medtronic Carelink 2490G

- Programming Header: Medtronic Vitatron 2067

- IPT coil: $16 \mathrm{~A}, 100 \mathrm{~W}, 6.78 \mathrm{MHz}$

- NARDA EHP200 Electric/Magnetic Field Analyzer

\section{B. Confirmed heating}

Exposing the pacemaker device to the EM field generated by the IPT coil showed no variation in the recorded temperature of the outer layer of both leads and pacemaker, demonstrating coherence with the results obtained in Section IV. Different orientation and distances of pacemaker body and lead were tested, and even in the worst case scenario (pacemaker along vertical axis of IPT coil at a distance of $12 \mathrm{~cm}$ ), no significant temperature variation was recorded. Fig. 3 shows thermal camera measurements performed before and after exposure.

\section{Variation of lead's impedance}

The conducted tests on lead impedance variation required the lead's electrode to be fixed to a tissue that emulates the myocardium impedance detected at the lead's end. Attempting to synthesize a tissue with similar properties through a variation of salinity of samples of agarose gel proved unfeasible. Despite obtaining realistic impedance values with $10 \%$ salinity weight to volume, the structural integrity of the material did not allow to fix the tip of the lead in a stable position, resulting in an inconsistent variation of the detected impedance.

More reliable results were obtained with the use of beef muscle $(255 \mathrm{~g}, \approx 2 \times 20 \times 7 \mathrm{~cm})$. Trying different probes separations led to baseline impedance values in a range of $300 \Omega$ to $800 \Omega$, which proved to be very close to the typical myocardium impedance detected during this type of measurements.

The lead impedance was measured in unipolar mode as shown in Fig. 4, with a $14 \mathrm{~cm}$ probe separation (which is a realistic scenario for pacemaker implants in children). The measurements were taken in two configurations: double looped lead and straight lead. Both cases ultimately led to the same results.

Different scenarios were considered to assess whether the impedance variation was actually due to a direct effect of the IPT-coil-generated magnetic field on the lead or due to the EMI caused to the interrogation device. When avoiding close proximity between the lead and the IPT coil while keeping the same distance between the coil and the reader, no impedance reading variation was recorded. This confirms that the detected impedance variation is actually due to the effect on the lead itself.

In Fig. 5 it is shown how the magnetic field generated ny the coil at a distance of $12 \mathrm{~cm}$ along the coil's vertical axis can temporarily affect impedance readings. The $738 \Omega$ baseline impedance showed a steady state variation of $40 \Omega$ during coil's operation. In [6] a permanent decrease in detected lead impedance can be an indication of early insulation damage, however the effect in this case is not permanent, as the impedance will revert to its baseline value after $40 \mathrm{~s}$ to $60 \mathrm{~s}$. The possible consequence of this phenomenon is a temporary oversensing of atrial readings.

The order of magnitude of impedance variation is however relatively small (approximately $5 \%$ of the baseline impedance), meaning that the effect of oversensing will be limited. Typical cases of permanent impedance variation due to lead damage are in the order of $30 \%$ to $40 \%$ [6], and even in the case they are detected they pose little to no danger to patients in the short term, indicating that phenomenon recorded during this experiment is not an indication of potential threats to pacemaker users.

\section{Interruption of Communication}

An interesting result observed is the effect on communication between the reader and the pacemaker.

The way a pacemaker device communicates with the reader is via wireless communication accessed through the activation 


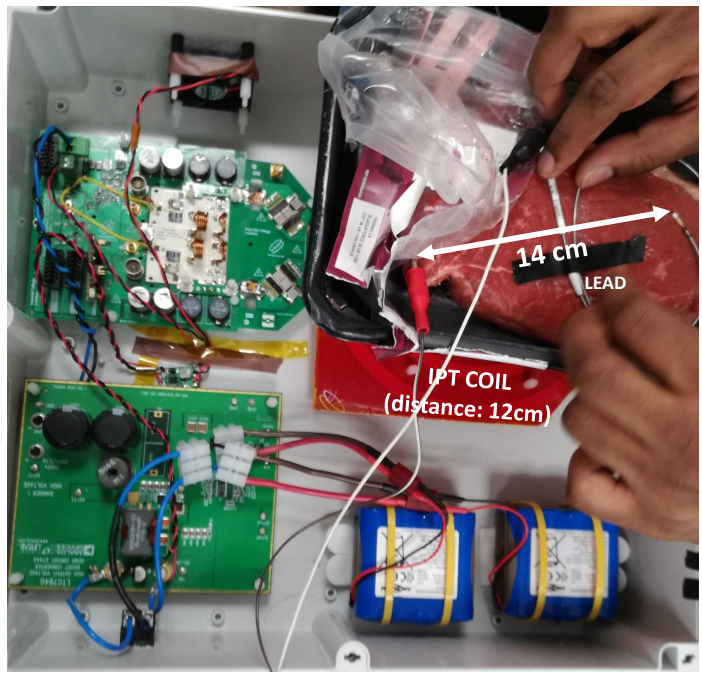

Fig. 4. Setup for measurements of lead's impedance

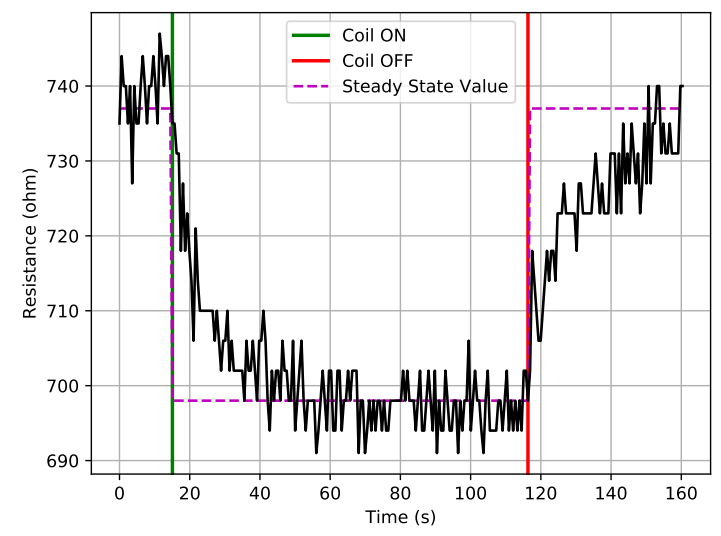

Fig. 5. Lead's impedance variation

of magnet mode. When the pacemaker enters this mode it starts pacing at a constant rate and it is possible to perform logging of the core events recorded by the device. Medtronic devices use MedRadio [7], a FSK digital communication type in the $401 \mathrm{MHz}$ to $406 \mathrm{MHz}$ band (with the core band being $402 \mathrm{MHz}$ to $405 \mathrm{MHz}$ ). Recent products are shifting to higher frequencies [8], implementing a communication type similar to BLE (Bluetooth Low Energy) in the $2.36 \mathrm{GHz}$ to $2.4 \mathrm{GHz}$ band.

When interrogating the device it was possible to notice an effect on the quality of the communication link. At distances below a $5 \mathrm{~cm}$ radius from the coil's centre we reported cases of complete interruption of communications.

This phenomenon is likely due to the interference caused on the FSK circuitry itself rather than interference on the transmitted signal as the output of the IPT coil is well outside the frequency band used for communication, both in the cases of standard MedRadio and BLE.

The effect produced poses no threats to the patient as it only affect the communication link at close distances, and the communication link itself does not affect pacing functionality.
TABLE I

COMPARISON WITH REPORTED CASES OF ASYNCHRONOUS MODE ACTIVATION

\begin{tabular}{ccc}
\hline \hline Technology & $\begin{array}{l}\text { Magnetic Field } \\
\text { recorded in } \\
\text { close proximity } \\
\text { (mT) }\end{array}$ & $\begin{array}{l}\text { Possible } \\
\text { Asynchronous } \\
\text { Pacing }\end{array}$ \\
\hline Radiofrequency Catether Ablation & 2 & $\checkmark$ \\
Magnetic Capsule Endoscopy & $200-1400$ & $\checkmark$ \\
Stereo Speakers with B $>10 \mathrm{G}$ & $>1$ & $\checkmark$ \\
Neodymium Magnets & $600-1400$ & $\checkmark$ \\
MRI & $200-7000$ & $\checkmark$ \\
Metal Detectors & $7.6 \mathrm{e}-3-0.37[11]$ & X \\
IPT system (in this work) & 0.1 & (X) \\
\hline
\end{tabular}

\section{E. Asynchronous pacing}

In order to avoid EMI-sensing related inhibition of pacing, asynchronous pacing is sometimes activated for MRI scans and surgery that requires use of diathermy (a surgical tool that produces EMI). This can be performed through a permanent magnet (typically $9 \mathrm{mT}$ ) placed in proximity of the device, allowing pacing at a fixed rate set by the programmer, regardless of any cardiac or other sensed activity. Although this mode can result in a fatal arrhythmia, it is not life threatening in the vast majority of the cases due to short duration and low pacing energy, but it can more commonly cause unpleasant symptoms of palpitations, breathlessness and neck pulsations due to ventricular activity being uncoordinated with atrial activity.

In [1] it is possible to observe that a wide range of technologies can lead to the possibility of triggering this mode as a consequence of exposure to strong EM fields. [9] and [10] debunked the potential triggering of Asynchronous Pacing in proximity of metal detectors and airport scanners highlighted in [1]. From a comparison of the other technologies reported (Table I) it is possible to estimate an approximation of the threshold for Asynchronous Mode triggering at around $1 \mathrm{mT}$. Although it is not ideal, it is not possible to directly assess whether this mode has been entered when performing invitro simulations. A technique to detect if this mode was entered would involve continuous ECG monitoring of a patient exposed to the analysed technology.

Fig. 6 shows the measured magnetic field in proximity of the IPT coil analysed. The magnetic field strength does not exceed $0.1 \mathrm{mT}$ even in close proximity of the coil along its vertical axis (where the field is higher). These measurements give an indication that triggering Asynchronous Mode is unlikely, as the magnetic field strength is more than ten times lower when compared to the cases reported in Table I.

\section{CONCLUSions}

The simulations and in-vitro tests conducted show good early stage results of $6.78 \mathrm{MHz}$ IPT systems posing no dangers for pacemaker users for power levels of up to $100 \mathrm{~W}$, while 


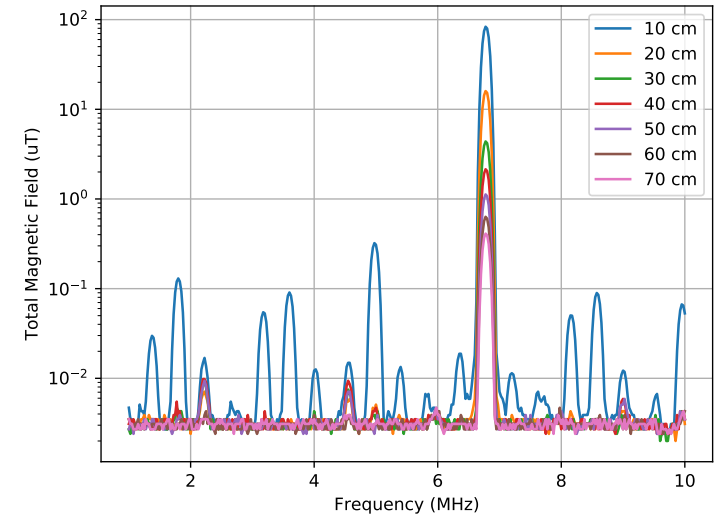

(a) Full Spectrum

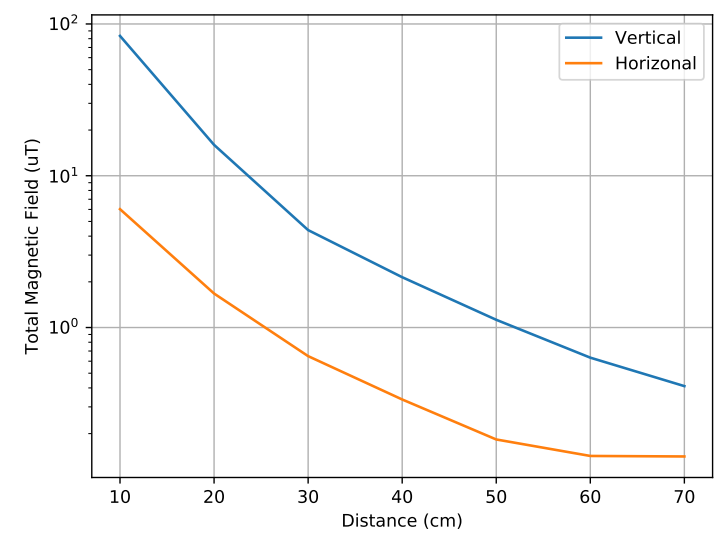

(b) $6.78 \mathrm{MHz}$ component

Fig. 6. Total Magnetic field varying distance on vertical axis of coil

still being conformal with the EN regulations [4] for device's safety with respect to temperature variations.

The observations drawn from variation of detected impedance and asynchronous mode's threshold estimations indicate that even in close proximity of a high-frequency IPT coil the direct effect on the patient would be minimum.

Despite the unfeasibility of a complete evaluation of the possible pacemaker effects through in-vitro testing only, these early stage results provide useful information about testing procedures and issues encountered, while giving early indica- tions of the safety of HF-IPT systems.

Further projects in collaboration with the National Heart \& Lung Institute, Imperial College London, U.K. have been planned in the near future, including feasibility of wirelessly charged devices in the field of medical technologies.

\section{ACKNOWLEDGEMENTS}

The authors would like to acknowledge the following funding source: EPSRC Impact Acceleration Account - Imperial College London 2017, grant ref: EP/R511547/1.

\section{REFERENCES}

[1] R. Beinart and S. Nazarian, Effects of external electrical and magnetic fields on pacemakers and defibrillators: From engineering principles to clinical practice. 2013.

[2] U. Lakshmanadoss, P. Chinnachamy, and J. Daubert, Electromagnetic interference of pacemakers. 2011.

[3] Kwan, C.H.; Yates, D.C.; Mitcheson P.D., "Design objectives and power limitations of human implantable wireless power transfer systems," Biological + Chemical Sensors Summit 2016: Implantable Biomedical Systems Symposium, 5-7 December 2016 (Invited Talk)

[4] BSI, BS EN 45502-1:2015, Implants for surgery. Active implantable medical devices. General requirements for safety, marking and for information to be provided by the manufacturer. June-2015.

[5] J. M. Arteaga, S. Aldhaher, G. Kkelis, C. Kwan, D. C. Yates, and P. D. Mitcheson, Dynamic Capabilities of Multi-MHz Inductive Power Transfer Systems Demonstrated with Batteryless Drones, IEEE Transactions on Power Electronics. pp. 11, 2018.

[6] Medtronic, Basic pacing concepts. Presentation slides available at https://www.slideshare.net/1171097100/basic-pacing-concepts/52 . January-2017.

[7] Federal Communications Commission. "Medical Device Radiocommunications Service (MedRadio)". https://www.fcc.gov/medical-deviceradiocommunications-service-medradio . 2017

[8] Versel, N. "FCC finalizes MBAN rule, still must appoint coordinator". MobiHealthNews. HIMSS Media. September 2012.

[9] Jilek C, Tzeis S, Vrazic H, Semmler V, Andrikopoulos G, Reents T, et al. Safety of Screening Procedures With Hand-Held Metal Detectors Among Patients With Implanted Cardiac Rhythm Devices: A Crosssectional Analysis. Ann Intern Med. 2011;155:587592

[10] Christof Kolb, Sebastian Schmieder, Gnter Lehmann, Bernhard Zrenner, Martin R Karch, Andreas Plewan, Claus Schmitt, Do airport metal detectors interfere with implantable pacemakers or cardioverterdefibrillators?, Journal of the American College of Cardiology, Volume 41, Issue 11, 2003, Pages 2054-2059, ISSN 0735-1097, https://doi.org/10.1016/S0735-1097(03)00424-8.

[11] Boivin, W.; Coletta, J.; Kerr, L. Characterization of the magnetic fields around walk-through and hand-held metal detectors. [Article] Health Physics. 84(5):582-593, May 2003. 